\title{
Dimensiones sociales de los riesgos tecnológicos: el caso de las antenas de telefonía móvil
}

\author{
Josep Espluga Trenc
}

Universitat Autònoma de Barcelona. Departament de Sociologia

08193 Bellaterra (Barcelona). Spain

joseplluis.espluga@uab.es

\section{Resumen}

La reciente proliferación de la telefonía móvil ha provocado un incremento de la preocupación pública por los posibles efectos de los campos electromagnéticos sobre la salud humana y sobre el medio ambiente. Aquí se presentan algunos de los resultados de una investigación exploratoria sobre las respuestas sociales a este riesgo tecnológico realizada en Cataluña. Se observa que, ni las medidas adoptadas por las empresas del sector (básicamente intentar ofrecer más información), ni las adoptadas por las administraciones públicas (básicamente generar normativas no siempre coordinadas entre si) sirvieron para mitigar el conflicto. Los resultados avalan algunos de los postulados de las recientes teorías sociales sobre la percepción social del riesgo (Wynne, etc.), en el sentido de que, en ocasiones, la oposición popular parece más relacionada con aspectos institucionales (quién gestiona el riesgo, cómo lo hace, etc.), que con los posibles daños a la salud, lo cual permite relacionar las respuestas sociales ante los riesgos tecnológicos con demandas de una gestión más transparente y/o participativa de dichos riesgos.

Palabras clave: percepción social, campos electromagnéticos, riesgo tecnológico, gestión del riesgo, participación ciudadana.

\section{Abstract. Social dimensions of technological risk: the case of mobile phone antennae}

The recent expansion of the mobile phone came together with the increase of public concerns related with perceived risks of electromagnetic fields to human health and environment. Here we show some of the main results of an exploratory research on the social responses to this technological risk in Catalonia. It can be said that neither the strategies implemented by companies (basically to offer more information to the public) nor the strategies developed by public institutions (to develop laws without coordination) were useful to manage the social conflict. This results support some findings of recent theories on social perception of risk (Wynne, etc.) showing that citizen protests against risk are based not only on the perception of health effects or harms, but also with other institutional risk dimensions (who and how manage the risk, etc.). This allows to link the social responses to technological risks with the need of a more participative and/or accountable risk management.

Key words: social perception, electromagnetic fields, technological risk, risk management, citizen participation. 


\section{Sumario}

\section{Introducción}

2. La percepción social del riesgo

3 . Unas notas de contexto sobre la introducción de la telefonía móvil en España

4. Respuesta de las empresas de telefonía móvil
5. Respuestas de las administraciones públicas

6. Las respuestas de la población afectada

7. Conclusiones. Implicaciones para la gestión de riesgos tecnológicos

Bibliografía

\section{Introducción}

La reciente proliferación de la telefonía móvil ha provocado, en España, como en otros países, un incremento de la preocupación pública por los posibles efectos de los campos electromagnéticos (EMF) sobre la salud humana. Esta preocupación ha llevado a muchos ciudadanos a expresar su rechazo contra la instalación de antenas de telefonía móvil en las cercanías de sus domicilios o de los colegios de sus hijos, y a exigir a los gobernantes la regulación de esta tecnología. Manifestaciones públicas ante los ayuntamientos o las sedes de las empresas operadoras, campañas de recogida de firmas a nivel de barrios, creación de plataformas ciudadanas y de asociaciones de afectados, organización de conferencias sobre los posibles efectos de los campos electromagnéticos sobre la salud, son algunas de las actividades que se han llevado a cabo en numerosas ciudades y pueblos para mostrar el rechazo hacia las antenas. A primera vista, todo parece indicar que las antenas de telefonía móvil se han conceptualizado en el imaginario popular como un potencial factor de riesgo.

El paradigma predominante de gestión del riesgo en nuestra sociedad se ha basado en una práctica combinación de ciencia y política: la utilización de evaluaciones del riesgo realizadas mediante métodos científicos, con la finalidad de obtener los datos más objetivos posibles, que posteriormente servirán de base a la toma de decisiones (política). Pero, al igual que tantas otras instituciones heredadas de la sociedad industrial clásica, la distinción entre la fase científica y la fase política de la gestión de riesgos está cada vez más cuestionada, especialmente en aquellos riesgos cuyo estudio cuenta con elevados márgenes de incertidumbre científica, tanto respecto a su naturaleza como a sus posibles efectos sobre la salud o sobre el medio ambiente. Como apuntan López Cerezo y Luján (2000), buena parte de las controversias académicas contemporáneas alrededor del riesgo pueden interpretarse como producto de las diferentes respuestas que se dan a la cuestión de si la evaluación de riesgos es separable, conceptual e institucionalmente, de las cuestiones éticas, sociales y políticas referentes a cómo el riesgo debe ser gestionado.

$\mathrm{Al}$ igual que en el caso de otros factores de riesgo contemporáneos, la investigación científica sobre los posibles riesgos derivados de los campos electromagnéticos (CEM) de la telefonía móvil presenta aún amplios grados de incertidumbre, de tal manera que hasta el momento sólo se ha podido demostrar 
la aparición de algunos daños en casos de exposiciones intensas, pero a penas hay evidencias en las situaciones de exposición ambiental de baja intensidad como las que se dan en la mayor parte de la vida cotidiana de las personas (Vecchia, 2001). Los efectos más visibles de los CEM sobre la salud son los efectos térmicos, y se ha comprobado que se manifiestan sólo cuando se superan determinados niveles de exposición y que dependen de la frecuencia que tenga el CEM en cuestión. De hecho, a causa de su relativa facilidad de identificación y de cuantificación, los efectos térmicos han sido utilizados por la Comisión Internacional para la Protección contra las Radiaciones No-Ionizantes (ICNIRP) como base para sus recomendaciones de seguridad. Unas recomendaciones que, a su vez, han sido utilizadas para elaborar la Recomendación del Consejo Europeo sobre la limitación de la exposición del público en general a los CEM (EUC, 1999). Más complicada es, en cambio, la detección de los posibles efectos a largo plazo de una exposición crónica a CEM, incluso de baja intensidad. Los investigadores tienden a coincidir en que este tipo de efectos no agudos, como cánceres, daños al ADN o alteraciones cognitivas, no se pueden desestimar a priori, sino que será necesaria una investigación más larga e intensa. En definitiva, la característica distintiva de los CEM respecto a otros factores tecnológicos "clásicos» radica en el grado de incertidumbre existente (al menos de momento) sobre sus posibles riesgos, tanto a la hora de probar que causen realmente daños a la salud (estudios epidemiológicos y de laboratorio), como a la hora de explicar cómo pueden suceder (encontrar un mecanismo plausible que conecte los CEM con la aparición de enfermedades). No obstante, los decisores (gobiernos, empresas) se ven empujados a regular esta tecnología por una opinión pública cada vez más suspicaz, debiendo tomar decisiones en condiciones de alta incertidumbre científica con la presión añadida de un hipotético amplio alcance de los daños futuros. Se trata de una situación que Funtowicz y Ravetz (1992) clasificarían como de ciencia postnormal.

Este texto presenta algunos de los resultados de una investigación exploratoria sobre las respuestas sociales a las antenas de telefonía móvil en Cataluña. Se trata de una investigación encargada por el Instituto de Estudios de la Seguridad ${ }^{1}$. Por ello, realizamos un análisis de contenido de los principales documentos generados por los principales actores sociales implicados en el conflicto, así como una serie de entrevistas en profundidad a personas clave ${ }^{2}$ relacionadas con estos actores sociales. En conjunto, se trata de una investigación con ánimo exploratorio que trata de describir la evolución de la problemáti-

1. El Instituto de Estudios de la Seguridad (IDES) es una iniciativa del Colegio de Ingenieros Técnicos Industriales de Barcelona y del Colegio Oficial de Ingenieros Industriales de Cataluña, que nace en 1998 como una fundación con la finalidad de estimular y difundir, desde una perspectiva transdisciplinaria, las problemáticas sociales bajo el ámbito conceptual de la seguridad. Una versión preliminar de algunos de los datos de esta investigación apareció en el volumen Observatori del risc. Informe 2002, editado en catalán por el IDES.

2. Las entrevistas se realizaron a: representantes de empresas del sector (2), miembros de instituciones estatales (2), personas que se consideran afectadas por los CEM de las antenas de telefonía móvil (3), de las cuales, 2 formaban parte de asociaciones formales de afectados. 
ca, con la finalidad de generar hipótesis de trabajo para intentar entender los aparentemente extraños comportamientos de los diferentes actores sociales (empresas, administraciones públicas y ciudadanos) involucrados en este tema.

\section{La percepción social del riesgo}

Las investigaciones sobre la percepción del riesgo aparecen y se generalizan básicamente a partir de los años setenta del siglo XX y responden a la necesidad cada vez más urgente de comprender y mitigar las protestas ciudadanas hacia ciertas tecnologías o actividades industriales (energía nuclear, plantas químicas, vertederos de residuos, etc.). Ante esta situación de protestas, se consideró prioritario identificar la percepción del riesgo que tenían los individuos, para así poder tomar decisiones encaminadas a paliar el desfase existente entre éstos y los expertos. Ello propició la aparición del concepto de riesgo aceptable, que venía a indicar el umbral a partir del cual los individuos que hicieran un cálculo racional de sus costos y beneficios, dejarían de oponerse a aquellas instalaciones tecnológicas (Starr, 1969). Esta perspectiva fue rápidamente cuestionada, especialmente desde el ámbito de la psicología cognitiva, cosa que dio lugar a una serie de estrategias de investigación que posteriormente se conocerían como paradigma psicométrico. Por otro lado, durante los años ochenta del siglo XX, se desarrolla la investigación sobre comunicación del riesgo, ya que, a ojos de expertos y decisores (de empresas y políticos), parecía evidente que, para que la gente dejara de tener aquellas creencias y comportamientos «irracionales», se la había de informar y formar adecuadamente, para que entendiera la bondad de los análisis técnicos expertos de riesgos y así eliminar los sesgos cognitivos que tuviera.

Este tipo de intervenciones se han revelado útiles y necesarias, pero también claramente insuficientes, ya que numerosos estudios han constatado que las personas «bien informadas» no siempre actúan tal y como los expertos esperan o quisieran. En este sentido, por ejemplo, algunos autores han sugerido y comprobado que, muy a menudo, los comportamientos aparentemente «extraños» o «irracionales» de ciertos individuos o grupos tienen que ver más con las creencias y los valores culturales que comparten que con las capacidades de generar daño objetivamente existentes (Douglas y Wildavsky, 1982). Hasta los años noventa del siglo XX, no se empieza a poner mayor énfasis en perspectivas de carácter más integrador que tienen en cuenta el marco social, político y cultural en el que tienen lugar los procesos de percepción y de comunicación de riesgos. Así, por ejemplo, Wynne sugiere que las percepciones sociales del riesgo no están tan directamente relacionadas con percepciones o evaluaciones de alguna cosa objetivamente existente, sino más bien con las relaciones que las personas mantienen con las instituciones responsables de gestionar dicho riesgo. Según este autor, como en las estimaciones expertas del riesgo hay numerosos y elevados niveles de incertidumbre, es perfectamente racional que los individuos no se limiten a ellas a la hora de valorar las magnitudes de los riesgos. Es lógico, pues, que se pregunten también sobre cosas como qué tipo de confianza les 
merecen las instituciones implicadas en la gestión del riesgo: «Las percepciones públicas y las respuestas al riesgo están basadas en juicios racionales sobre la conducta de las instituciones expertas y sobre su capacidad para ser dignas de confianza» (Wynne, 1996: 57). En definitiva, las percepciones sobre el riesgo implican algún elemento de juicio sobre la calidad de las instituciones implicadas, por lo que cobran relevancia lo que Wynne llama las dimensiones institucionales del riesgo, es decir, dimensiones como la confianza que se puede tener en una institución (responsable de gestionar un riesgo), la existencia de relaciones de dependencia con dicha institución, su independencia (percibida) respecto a otros agentes sociales, la justicia percibida en sus actuaciones, su legitimidad (percibida), su competencia (percibida), etcétera.

Por otro lado, desde un punto de vista teórico, podemos considerar que en cualquier conflicto relacionado con riesgos ambientales se puede pensar en un sistema de interacciones compuesto por, como mínimo, tres tipos de actores principales (figura 1):

a) Los promotores o generadores del riesgo: que, en este caso, estarían representados por las empresas operadoras de telefonía móvil, que son las interesadas en promover y difundir esta tecnología y en obtener unos beneficios empresariales.

b) La población afectada: que, en este caso, serían las personas que se perciben perjudicadas por la instalación de las antenas, ya sea en sentido económico, ambiental, de salud, etc.

c) Los encargados de garantizar un nivel de seguridad: que, en este caso, son las instituciones públicas como la Administración central del Estado, la Administración autonómica y las administraciones locales y los ayuntamientos.

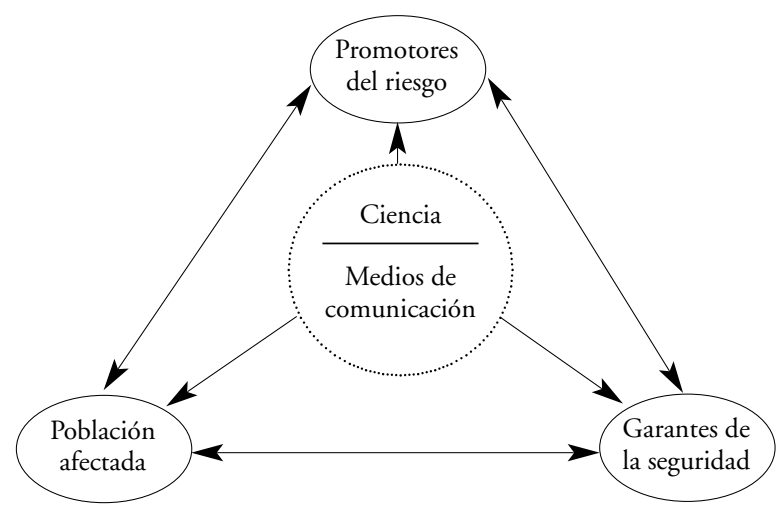

Figura 1. Sistema de interacción típico de los conflictos sociales relacionados con riesgos tecnológicos. 
Resulta evidente que estos actores sociales no siempre tienen una estructura unitaria, sino que, dentro de cada uno de ellos, se pueden distinguir intereses y coyunturas diferentes, si bien, en general, se encuentran situados en posiciones similares respecto a la estructura del conflicto. Además, a estos tres actores principales habría que añadir otro que, en el tema que nos ocupa, desempeña un papel fundamental: la ciencia. Los expertos científicos pueden aparecer vinculados (proveyendo conocimientos o legitimidad) a cualquiera de los anteriores actores, por eso los mencionamos a parte. También los medios de comunicación social se podrían considerar integrantes de este marco de interrelaciones, en su rol de impulsores, amplificadores o mitigadores de los conflictos ambientales o relacionados con riesgos tecnológicos. Los medios de comunicación social hacen visibles los riesgos invisibles y proporcionan a los individuos un conocimiento que les permite relacionar unos factores de riesgo con unos efectos o daños, con lo que contribuyen también a definir el riesgo. Al igual que los científicos, los medios de comunicación pueden aparecer vinculados a cualquiera de los tres actores principales.

\section{Unas notas de contexto sobre la introducción de la telefonía móvil en España}

A pesar de que la telefonía móvil empieza a funcionar en España a principios de los años noventa, es hacia 1995, con la liberalización del mercado de las telecomunicaciones (que rompe el antiguo monopolio estatal), cuando se genera un rápido crecimiento de las infraestructuras necesarias para ofrecer los servicios telefónicos por parte de nuevas empresas operadoras. En un mercado virgen, tener más antenas instaladas significaba poder captar más clientes, por lo que estas empresas mantenían entre sí relaciones de dura competencia comercial. Además, las comunicaciones móviles son un importante sector estratégico desde el punto de vista político y económico, y las licencias adjudicadas en concurso por el Gobierno español obligaban a las empresas operadoras a ofrecer unos servicios mínimos de cobertura en un plazo de tiempo relativamente breve, lo que supuso una presión añadida al despliegue de la red de infraestructuras y antenas. Así, primero fue Telefónica Móviles la empresa que se lanzó a instalar antenas por todo el territorio (el grueso de su despliegue tuvo lugar entre mediados de 1995 y finales de 2000), después Airtel (que más tarde mutaría en Vodafone) (sobre todo entre octubre de 1995 y 1999) y más tarde una tercera empresa, Amena (se desplegó con intensidad entre enero de 1999 y noviembre de 2000). Y aún hay una cuarta empresa con licencia para operar con telefonía móvil con tecnología UMTS, Xfera, la cual, cuando se hizo la presente investigación, aún no había empezado a emitir (aunque intentó iniciar el despliegue de sus antenas hacia enero de 2001). La dura competencia y desigualdad inicial entre estas empresas comportó que, a la hora de instalar las antenas sobre el territorio, no repararan en medios y no siempre actuaran dentro de la legalidad. Así, con frecuencia, especialmente en los primeros años, se instalaban antenas sin los preceptivos permisos municipales o sin informar 
al vecindario (tal y como han reconocido diversas de las personas entrevistadas, tanto de las propias empresas como de las administraciones públicas). Es en este contexto cuando, a finales de la década de los noventa, se registra un creciente movimiento ciudadano de protesta contra la instalación de antenas en lugares habitados ${ }^{3}$. Un movimiento normalmente poco organizado, formado mayoritariamente por personas que se movilizan en momentos puntuales, que se adhieren a manifestaciones populares que tienen lugar en sus lugares de residencia (barrios, pueblos, ciudades) y que fluctúa según la masa crítica que se genera en cada momento por motivos diversos no siempre previsibles (influencia mediática, etc.).

\section{Respuesta de las empresas de telefonía móvil}

Desde las operadoras de telefonía móvil (y del resto de empresas relacionadas con el sector), se contempló con preocupación la creciente alarma ciudadana y sus negativas consecuencias sobre sus planes de desarrollo empresarial. Ante la ausencia de normativa legal para el conjunto de España, todas las empresas operadoras elaboraron y aprobaron conjuntamente, en julio de 2001, un código de autorregulación que se abría con la siguiente frase: «Ante el estado de opinión creado respecto a las infraestructuras de telefonía móvil, los operadores que ofrecen este servicio en España adquieren el compromiso de autorregular sus actividades en el despliegue de la red. El presente documento recoge el compromiso que los operadores de telefonía móvil asumen en el despliegue de la red con el objetivo de garantizar la salud de los ciudadanos» (ANIEL, 2001). Este código de autorregulación y buenas prácticas incluyó restricciones a las emisiones radioeléctricas y a los límites de exposición, así como la solicitud de evaluación sanitaria periódica de las fuentes de emisión de CEM a la Administración pública. Como se puede observar, se trató de un intento de generar confianza entre la población y de dar una imagen de transparencia en cuanto a la información sobre los posibles riesgos y daños a la salud.

La perspectiva de las empresas operadoras también la encontramos en un monográfico de la revista Electrónica \& Telecomunicaciones (revista de la Asociación Nacional de Industrias Electrónicas y de Telecomunicaciones), aparecido en el número 11 (febrero de 2002). Así, por ejemplo, en un extenso artículo titulado «El poderoso influjo de una sinrazón» (Barrio, 2002: 6-23), después de exculpar a «los ciudadanos que se preocupan por la salud de sus hijos

3. Este proceso parece tener un especial efecto amplificador del conflicto desencadenado por la aparición de varios casos de leucemia entre los niños de un colegio de Valladolid, en el año 2000, que las asociaciones de padres y madres de alumnos atribuyeron a las numerosas antenas de telefonía móvil existentes en el tejado de un edificio cercano a las aulas. La dimensión mediática que alcanzó el caso obligó al Ministerio de Sanidad a realizar un estudio sobre la posible influencia de las antenas en la aparición de las leucemias, sin resultados aparentes. 
o las comunidades de vecinos que persiguen el máximo beneficio económico por alquilar tejados», se exigen responsabilidades por la alarma creada básicamente a las administraciones públicas y a los medios de comunicación. A las primeras, por no haber sabido explicar la pertinencia de las normas legales vigentes y, en el caso de las administraciones autonómicas y locales, por haber impuesto demasiadas restricciones basadas en "criterios puramente políticos». $\mathrm{Y}$, a los segundos, por parcelar las noticias y presentarlas con un sesgo sensacionalista o sin el rigor que requieren las "cuestiones socialmente sensibles». De todas formas, hay que apuntar que este diagnóstico desde el sector tampoco está libre de sesgos y parece más bien una reinterpretación ad hoc para justificar a posteriori la evolución que ha seguido la problemática. De acuerdo con los datos disponibles, es dudoso que las propias empresas del sector calificaran desde el principio la percepción social de los riesgos de las antenas de telefonía móvil como «cuestiones socialmente sensibles». Una de las personas entrevistadas, responsable de una empresa del sector, hace una valoración sobre cómo han ido las cosas y reconoce que la alarma social cogió por sorpresa a las propias operadoras.

En un inicio, las operadoras no le atribuimos la importancia que se le tenía que haber atribuido. Lo vimos como un problema secundario y quizá no pusimos hilo en la aguja a nivel de contrarrestar esta alarma social con información fiable para la población... de llevar a cabo acciones un poco... para apaciguar esta alarma social, que tranquilizasen a la opinión pública. (Empresa A)

Desde el punto de vista del sector, siguiendo con el discurso expresado en el informe mencionado anteriormente (Barrio, 2002: 11), se tiende a considerar que «buena parte de la actual polémica está basada en la confusión entre dos tipos de emisiones [las radiaciones ionizantes y las no ionizantes]». Es decir, se sugiere que hay una falta general de información esencial entre las personas que rechazan las antenas en determinados emplazamientos, que confunden radiación con radioactividad.

De acuerdo con estas interpretaciones por parte de los responsables de empresas operadoras, una de las propuestas que realizan para reducir la respuesta social en un futuro es intentar evitar o minimizar el impacto visual de las antenas, con el objetivo de hacerlas pasar desapercibidas para la ciudadanía.

Nosotros tenemos comprobado que en aquellos lugares donde las antenas se ven mucho y son demasiado grandes... hay más protestas y más problemas de tipo psicosocial. Por eso pensamos que el mimetizarlas puede ser una vía para conseguir disminuir el rechazo. Y lo comprendo, eh. Porque hay algunas antenas que... que no veas... Pero eso también es cuestión de dinero y de tiempo, primero hemos de amortizar las actuales... (Empresa B)

Desde las operadoras, se acusa también a los medios de comunicación de publicar noticias poco contrastadas y de así predisponer al público en contra. 
Lo que nos ha hecho mucho daño es la publicación en la prensa de noticias en periódicos de mucha tirada, que hacen entrevistas a gente [...] de cosas que no son ciertas, y los periódicos lo publican como si fuera una verdad imperativa. Y claro, cuando una persona que tiene dudas lo lee... pues, claro... Entonces nosotros ya podemos contraatacar con mediciones objetivas y tal... que no hay nada que hacer... (Empresa B)

De cara al futuro, los responsables de las empresas operadoras expresan su deseo de que la situación se reconduzca, los conflictos se acaben y puedan continuar con sus planes de negocio. Así, las directrices principales de sus estrategias de futuro tienen que ver, sobre todo, con la transmisión al público de información de calidad, con recuperar las buenas relaciones con los ayuntamientos y con propiciar el fomento de la investigación sobre los efectos de los CEM para la salud (y anunciarlo públicamente).

\section{Respuestas de las administraciones públicas}

A un nivel supraestatal, el Consejo de Ministros de Sanidad de la Unión Europea publicó, el 12 de julio de 1999 (EUC, 1999), un informe relativo a la exposición del público en general a campos electromagnéticos (CEM), donde se incluyen los campos estáticos, los campos de extremada baja frecuencia (ELF) y los campos de radiofrecuencia (RF), que abarcan la gama de frecuencias de 0 hercios a 300 gigaherzios. En este informe, se recomendaba a los estados miembros de la Unión Europea la adopción de un marco de restricciones básicas y de niveles de referencia, basados en los pocos efectos para la salud bien estudiados (los efectos térmicos) relativamente fáciles de evaluar en la práctica.

En España, en el año 2000, la Subdirección General de Sanidad Ambiental y Salud Laboral (Dirección General de Salud Pública y Consumo, del Ministerio de Salud y Consumo) convocó un comité pluridisciplinario de científicos independientes para que realizaran una revisión exhaustiva de la bibliografía epidemiológica internacional y redactaran un informe técnico sobre los CEM y su posible incidencia en la salud pública. Este comité de expertos concluyó que, según los conocimientos científicos disponibles, se puede afirmar que la exposición a campos electromagnéticos no ocasiona efectos adversos para la salud, dentro de los límites establecidos en la recomendación del Consejo de Ministros de Sanidad de la Unión Europea (1999/519/CE), y que, por tanto, su cumplimiento debía bastar para garantizar que las antenas de telefonía y los terminales móviles no representaran ningún peligro para la salud pública. No obstante, añadían que «en cumplimiento con el principio de precaución, y a pesar de la ausencia de indicios de efectos nocivos para la salud, conviene fomentar el control sanitario y la vigilancia epidemiológica, con la finalidad de hacer un seguimiento a medio y largo plazo de las exposiciones a campos electromagnéticos» (MSC, 2001).

En septiembre de 2001, el Gobierno español (a través del Ministerio de Sanidad y Consumo) desarrolló un marco legal para ordenar las infraestruc- 
turas de telefonía móvil (Real Decreto 1066/2001, de 28 de septiembre, por el que se aprueba el Reglamento que establece las condiciones de protección del dominio público radioeléctrico, restricciones a las emisiones radiadas y medidas de protección ante emisiones radioeléctricas). Esta normativa reguló la autorización, la planificación y la inspección de instalaciones radioeléctricas, fijó unos límites de exposición (que fueron los mismos de la recomendación del Consejo Europeo de 1999) y exigió un estudio detallado de los niveles de exposición radioeléctrica para las nuevas peticiones de autorización de instalaciones de antenas.

En el caso concreto de Cataluña, en mayo de 2001, cuatro meses antes de que apareciera la legislación impulsada por el gobierno central, el gobierno autónomo catalán aprobó una normativa sobre el mismo tema (Decret 148/2001, de 29 de maig, d'ordenació ambiental de les instal.lacions de telefonia mòbil i altres instal-lacions de radiocomunicació), con la finalidad de regular las instalaciones de radiocomunicación susceptibles de generar campos electromagnéticos que se emplacen en su territorio. Mediante este decreto, el Gobierno catalán fijó unos niveles de referencia y unas distancias de protección a las personas superiores a los de la Recomendación de la Unión Europea y, por tanto, superiores también a las que unos meses después fijaría el Gobierno español. También estableció un sistema de ordenación urbanística de los emplazamientos de nuevas antenas y el requerimiento de un permiso municipal (una «licencia ambiental») para cada nueva antena. Finalmente, propuso que los ayuntamientos pudieran también aumentar la regulación de algunos aspectos relativos a la telefonía móvil, de acuerdo con las competencias municipales sobre protección de la población, salud, medio ambiente, urbanismo y vivienda.

El caso es que antes del 2001, mientras todavía no existía ninguna normativa estatal ni autonómica que regulara este tema, y presionados por las crecientes movilizaciones ciudadanas, muchos ayuntamientos ya habían decidido llevar a cabo acciones para dar alguna respuesta a los conflictos sociales que florecían en sus municipios. A grandes rasgos, y de manera similar a otros lugares de España, en Cataluña se dieron tres tipos de respuestas:

- La mayoría de los ayuntamientos decidieron aplicar una moratoria indefinida a la concesión de licencias para nuevas antenas, mientras ganaban tiempo para que la situación se clarificara (es el caso de municipios como Terrassa, l'Hospitalet de Llobregat o Montcada i Reixach, por ejemplo).

- Otros ayuntamientos regularon los procedimientos para otorgar licencias para nuevas antenas mediante una normativa propia (ordenanzas municipales), pero dejando la fijación de unos valores límite de referencia a la «normativa que establezca la Administración competente» (es el caso, por ejemplo, de Santa Coloma de Gramenet, Mollet o Barcelona).

- Por último, algunos (pocos) ayuntamientos que tenían una política municipal clara al respecto, establecieron por su cuenta unos valores límite y distancias de seguridad mediante una normativa propia (por ejemplo, Sant Feliu de Guíxols). 
En conjunto, se configuró un mapa territorial muy complejo, donde los criterios de seguridad variaban enormemente de un municipio a otro. Las disposiciones legales promovidas por los gobiernos catalán (mayo de 2001) y español (septiembre de 2001), adoptaron criterios diferentes y sólo establecieron unas normas de mínimos, dejando a cada ayuntamiento la posibilidad de incrementarlos por su cuenta, con lo que se consiguió aumentar aún más la diversidad de regulaciones (y la confusión). Como resultado, dos años después, algunos ayuntamientos aún mantenían la moratoria, mientras que muchos otros impusieron a las empresas de telefonía móvil unas condiciones más duras y dispares para la instalación de nuevas antenas, más basadas en criterios políticos que científicos, con lo cual, a menudo, las distancias obligatorias o intensidades de potencia que son consideradas legales en un municipio, no lo son en el municipio vecino. En definitiva, la existencia de un marco legal no desactivó el conflicto social, entre otras cosas porque su diversidad y disparidad de criterios ha podido contribuir a reafirmar la desconfianza de los ciudadanos. $\mathrm{Y}$, además, este marco legal tan fragmentado ha venido a complicar mucho más los planes de expansión de las empresas operadoras.

\section{Las respuestas de la población afectada}

En primer lugar, hay que apuntar que la telefonía móvil no es una tecnología extraña para la población estudiada, muy al contrario, se trata de un servicio ampliamente utilizado, incluso por aquellas personas que manifiestan su preocupación por la presencia de las antenas ${ }^{4}$. Pero tal cosa no impide que estas mismas personas reclamen un cambio del modelo de gestión de esta tecnología (y no sólo la simple eliminación de las antenas de las ciudades). La investigación exploratoria efectuada muestra que, en un momento dado, las personas que estaban mínimamente preocupadas por los efectos de los CEM, comenzaron a buscar información por su cuenta (en especial a través de Internet), lo cual implica un grado de esfuerzo notable, ya que, como los mismos afectados reconocen, la información disponible es muy heterogénea y no siempre resulta fácil orientarse.

La información viene a partir de periódicos, de cosas que vas leyendo, comentas un día algo a algún vecino, o algún pariente te va pasando recortes de periódico, [...] finalmente acabas consiguiendo unas webs y miras lo que hay por allí, haces una cierta selección, intentas... pues... discriminar un poco lo que encuentras, o escoger un poco, porque encuentras desde posturas apocalípticas que dicen que acabaremos todos enfermos, a otras que dicen que es abso-

4. En el momento en que se produce la problemática aquí explicada (2001), el 64,2\% de los hogares de Cataluña disponían de teléfono móvil, con un total de casi dos millones y medio de unidades, lo que representaba una media de 1,2 móviles por cada hogar de Cataluña (OSI, 2001). Esta misma fuente nos dice que en Cataluña había, el 1 de abril de 2001, 4,7 millones de líneas activas de telefonía móvil. Además, se trata de unos datos con una fuerte tendencia al alza. 
lutamente inocuo... (Afectado A, miembro de una asociación contra las antenas)

Esta búsqueda individual de información contribuyó a poner en contacto a personas inquietas, y propició la formación de grupos más o menos fluctuantes de afectados. En este proceso, debió ser importante la influencia de ciertas personas que actuaron a modo de estaciones amplificadoras del riesgo (Pidgeon y otros, 2003) y que contagiaron su preocupación y sus puntos de vista a las personas de su entorno social. Se observa que las personas más activas y movilizadas contra las antenas son también las que más información han buscado y han encontrado. Desde este punto de vista, se podría poner en duda la eficacia de la estrategia propuesta por administraciones públicas y empresas operadoras, basada principalmente en ofrecer información «clara» al público, ya que estas personas han aprendido a seleccionar sus fuentes y ya se consideran a sí mismas suficientemente bien informadas.

No obstante, la estrategia de dar información «clara» sí que puede servir para "convencer» a la gente que todavía no se ha preocupado demasiado ni se ha movilizado por el tema.

Mira, de momento, me gustaría [tener información] incluso por curiosidad, saber qué es lo que emiten, y si están por debajo de los límites que recomiendan las normativas o... Y bueno, yo no voy a defender que las quiten, porque soy una consumidora de móvil a más no poder. Por tanto... (Afectada C)

Uno de los aspectos más reveladores de este conflicto ha sido contrastar la hipótesis de Wynne (1992, 1996), según la cual, la percepción pública del riesgo no se centra sólo en los posibles daños a la salud, sino que hay otras dimensiones que los ciudadanos tienen muy en cuenta a la hora de decidir sus comportamientos contrarios (o no) a las antenas. En las entrevistas, aparecen otras dimensiones de consecuencias del riesgo, como daños o pérdidas económicas, de calidad de vida, estéticas o paisajísticas, sentimientos de injusticia, de agresión a su dignidad, etc. Unas dimensiones que los gestores del riesgo a menudo parecen ignorar o no detectar. Uno de los afectados lo explica muy bien:

Hombre, para oponerse hay motivos. La salud me parece lo fundamental, pero hay otros, a nivel por ejemplo paisajístico, de paisaje urbano. Es lo que te decía, cuando llegué de las vacaciones en septiembre y me encontré la antena... pensé «vaya». Eso por un lado, y si tú te compras un piso y no tiene una antena ahí delante, y ahora ya la tiene, pues ya no es el piso que yo compré. Después está el problema de la devaluación de los pisos, que es un problema que... que hay gente a la que le da un poco de vergüenza tocar, que dicen "oh, que el tema es la salud", pero también es estas otras cosas. (Entrevistado A, miembro de una asociación contra las antenas)

Además, se observa que, en la percepción de este riesgo, también influye la visión que muchos ciudadanos tienen de las empresas operadoras y de sus 
prácticas a la hora de emplazar las antenas, en ocasiones percibidas como un tanto oscuras. Estas prácticas poco transparentes han contribuido a crear una sensación de desconfianza, que se añade al sentimiento de imposición abusiva y no querida de aquel riesgo. Y se observa que tal percepción no se limita sólo a las actuaciones de algunas empresas instaladoras de antenas, sino también a las de algunas administraciones públicas (que no siempre efectúan su tarea inspectora con la eficacia deseable).

Las personas que todavía no están demasiado movilizadas contra las antenas tienden a reclamar una información clara y transparente, que les permita mantener la confianza en las instituciones y en las empresas gestoras del riesgo. En cambio, las reivindicaciones de las personas más movilizadas y activas van encaminadas a exigir un nuevo modelo de organización de la telefonía móvil, que tenga en cuenta las actuales incertidumbres científicas respecto a temas de salud y, muy especialmente, que tenga más en cuenta a los ciudadanos (sus intereses, sus formas de vida, etc.) a la hora de distribuir las infraestructuras tecnológicas por el territorio. Ante las dificultades para incidir y participar en las decisiones sobre los emplazamientos y sobre los avances tecnológicos, hoy por hoy, los afectados parece que prefieren argumentar sus resistencias apelando a la salud, pero tal apelación puede que sólo sea un indicador de un iceberg oculto de reclamación de mayor participación ciudadana en ámbitos que hasta ahora se han venido gestionando de forma eminentemente tecnocrática.

\section{Conclusiones. Implicaciones para la gestión de riesgos tecnológicos}

A nuestro entender, el caso de las respuestas sociales a las antenas de telefonía móvil permite ilustrar con claridad los límites del modelo de gestión de riesgos predominante en nuestra sociedad. Veamos algunos de los más relevantes:

a) Las limitaciones de la estrategia de la información. Para intentar desactivar las protestas ciudadanas contra las antenas de telefonía móvil, las empresas del sector supusieron que lo que necesitaba la gente era simplemente más información sobre los CEM y sus riesgos (o su ausencia de), para lo cual implementaron varias campañas informativas. Pero esta estrategia, aunque necesaria, por sí sola tiene una eficacia más que discutible para mitigar el conflicto social abierto. Las personas que perciben las antenas de telefonía móvil como factores de riesgo no constituyen un conjunto homogéneo, sino que, como mínimo, se pueden dividir en dos tipos: por un lado, aquéllas que todavía no tienen una expectativa clara y para quienes dichos riesgos no constituyen una prioridad, y, por otro lado, aquéllas preocupadas que, después de un proceso de reflexión más o menos intenso, se han movilizado activamente en contra de las antenas (e incluso han constituido grupos organizados y más o menos estables de oposición a las mismas). El análisis de las entrevistas realizadas indica que, mientras que las primeras estan predispuestas a recibir nueva información "clarificadora» 
sobre los riesgos, las segundas se muestran reticentes a aceptar ningún tipo de información procedente de unas empresas e instituciones en las que no confían (de hecho, se consideran a sí mismas ya muy «bien informadas»). Desde este punto de vista, la clásica estrategia de la comunicación unidireccional de riesgos no parece que pueda servir para disminuir este conflicto social. Es más, puede incluso contribuir a exaltar los ánimos de esta población que ya se cree bien informada y que ha aprendido a filtrar sus fuentes, porque puede interpretar el proceso de comunicación como una simple estrategia manipuladora. En este sentido, y teniendo en cuenta que, en todo proceso de gestión de riesgos tecnológicos, será preciso difundir una información clara y transparente, el caso de la respuesta social a las antenas de telefonía móvil pone en cuestión el modelo de comunicación unidireccional (de expertos a legos) llevado a cabo por empresas y administraciones públicas. $\mathrm{O}$, al menos, problematiza el tempo con el que se ha desarrollado.

b) El papel de las administraciones públicas. A causa de su proximidad a los ciudadanos, los ayuntamientos fueron las primeras administraciones públicas que llevaron a cabo acciones precautorias (moratorias, restricciones, etc.), pero de manera bastante descoordinada. El hecho de que, en una primera fase, no se supiera dar una respuesta inmediata a las demandas ciudadanas, pudo deteriorar la confianza pública en aquellas instituciones, con lo que dejaron de ser interlocutores válidos para aquellos ciudadanos. Cuando el conflicto social se expandió, otras administraciones públicas de nivel territorial superior (nacionales y autonómicas) promulgaron legislaciones específicas, pero también con criterios dispares, con lo que la confusión se mantuvo. Así, es muy significativo el hecho de que el aumento unilateral de las restricciones a la instalación de antenas por parte de algunas administraciones públicas (ayuntamientos, Generalitat, etc.), a pesar de que se hizo con la finalidad de garantizar una mayor seguridad, no consiguieran tal objetivo en términos de percepción del riesgo. Más bien al contrario, la percepción del riesgo se sobredimensionó. En cierta manera, los aumentos unilaterales de restricciones aprobados por la Generalitat y por muchos ayuntamientos respecto al Decreto del Ministerio de Sanidad y Consumo de septiembre de 2001, contribuyeron a desautorizar las recomendaciones de referencia de las instituciones expertas (comité de sabios convocado por el propio Ministerio, Unión Europea, OMS, etc.). Porque, si la Unión Europea dice que a un nivel $\mathrm{X}$ difícilmente ocurrirán daños a la salud, ¿por qué aumentar el nivel a $\mathrm{X}+n$ ? Y si se hace esto, ¿por qué no aumentar la restricción hasta $\mathrm{X}+n+n$ ? Probablemente hubiera sido más razonable justificar el aumento de las restricciones simplemente como resultado de una negociación implícita entre las partes afectadas (como, de hecho, sucedió), aunque reconocer tal cosa es poco compatible con el modelo hegemónico de gestión del riesgo.

c) Las dimensiones sociales e institucionales del riesgo. Este caso muestra que las respuestas ciudadanas tienen que ver sólo en parte con los temores de daños 
a la salud, que también existen (se observa, por ejemplo, que algunas personas parecen desarrollar una hipersensibilidad fisiológica a los CEM). Más allá de efectos sobre la salud, hay quienes se oponen a las antenas porque se han sentido menospreciados o injustamente tratados por las empresas instaladoras (que han situado la antena ante su domicilio ilegalmente o apresuradamente y sin dar ningún tipo de explicación) o por las administraciones públicas (que han vulnerado ciertos derechos o han permitido prácticas percibidas como poco honestas por parte de ciertos ciudadanos). O porque la antena comporta un impacto paisajístico que les lleva a considerar que aquél ya no es el piso que compraron, o creen que su propiedad inmobiliaria se devaluará en el mercado a causa de la cercanía a la antena. Estas otras dimensiones de daños o pérdidas a consecuencia del riesgo raramente son tenidas en cuenta en los procesos de evaluación de riesgos, una exclusión que contribuye a que estos fenómenos se presenten como ingobernables ante los gestores del riesgo (o como sólo gobernables mediante procedimientos autoritarios). Aunque hay que reconocer que incluir estas dimensiones en las evaluaciones del riesgo requeriría generar nuevas metodologías de análisis capaces de incorporar puntos de vista de todos los actores implicados, lo cual, a su vez, comportaria reformas institucionales de gran calado.

d) El modelo tecnocrático de evaluación del riesgo. Desde las administraciones públicas, se justifica la descoordinación inicial apelando a la incertidumbre que transmiten los científicos que estudian el tema. Este argumento hay que entenderlo en función del modelo de gestión del riesgo predominante en las instituciones públicas (de orientación eminentemente tecnocrática), donde este tipo de decisiones se toman de acuerdo con unos conocimientos expertos que constituyen el corpus de datos «objetivos» sobre los que los políticos deciden. Pero ante una problemática como la de los CEM, donde la propia ciencia reconoce enfrentarse a dificultades enormes y no disponer todavía de suficientes evidencias para garantizar su inocuidad, y donde, además, es necesario tomar decisiones rápidas y de gran importancia estratégica económica y política, aquella lógica tecnocrática muestra sus limitaciones. Desde este punto de vista, los conflictos ambientales pueden entenderse también como una demanda de cambio del modelo de toma de decisiones sobre aspectos públicos.

e) Déficits democráticos en la toma de decisiones. En definitiva, en Cataluña, como en el resto de España, muchos ayuntamientos han aplicado diversas modalidades del principio de precaución, en forma de moratorias, de restricciones a las nuevas antenas, etc., como una forma de gestionar los conflictos sociales motivados por la instalación de antenas de telefonía móvil. A pesar de que estas restricciones y moratorias se han argumentado como una forma de proteger la salud de la población de los riesgos de los CEM, en la práctica han respondido a una multitud de factores diferentes, como los mencionados anteriormente, a los que cabría añadir que los ayuntamientos son más vulnerables que otras administraciones a la presión ciu- 
dadana. Así, muchos políticos locales no han querido quedar mal ante su población (electorado) y parecen haber vivido la presión ciudadana como una amenaza a su estatus político. En cualquier caso, detrás de este conflicto social, a pesar de que se expresa en forma de reclamaciones por riesgos para la salud, parece haber una demanda de mayor participación ciudadana (o quizá tan sólo de más transparencia) en la toma de decisiones sobre este tipo de infraestructuras tecnológicas. Desde esta perspectiva, el hecho de que las administraciones públicas locales establezcan unos límites al despliegue de antenas con criterios más políticos que científicos, no tiene por qué ser negativo. En muchos casos, puede ser un indicio de que reconocen que los ciudadanos que se sienten afectados son también actores importantes en el juego político local.

\section{Bibliografía}

ANiEL (Asociación Nacional DE Industrias Electrónicas Y DE Telecomunicaciones) (2001). Código de Autorregulación de los operadores de Telefonía Móvil para el Despliegue de la Red necesaria para el Servicio. Madrid: ANIEL.

BARRIO, P. (2002). «El poderoso influjo de una sinrazón. ¿¿Efectos nocivos de la telefonía móvil?». Electrónica y Telecomunicaciones. Madrid, núm. 11, p. 6-23.

BergQvist, U.; Vogel, E. (eds.) (1997). Possible Health Implications of Subjective Symptoms and Electromagnetic Fields. A Report Prepared by a European Group of Experts for the European Commission, $D G V$. Arbete och Hälsa.

EsPLUGA, J. (2002). «Fenòmens NIMBY: Respostes socials a les antenes de telefonia mòbil». Observatori del risc. Informe 2002. Barcelona: Beta-IDES, p. 139-177.

EUC (EUROPEAN UNION COUNCIL) (1999). "Council Recommendation of 12 July 1999 on the limitation of exposure of the general public to electromagnetic fields $(0 \mathrm{~Hz}$ to $300 \mathrm{GHz}) »$. Official Journal of the European Communities, vol. L 199 (3007-1999), p. 59-70.

Fischhoff, B.; Slovic, P.; Lichtenstein, S.; Read, S.; Coombs, B. (1978). «How Safe is Safe Enough: A Psychometric Study of Attitudes towards Technological Risk and Benefits». Policy Sciences, núm. 8: 127-152.

Funtowicz, S.; Ravetz, J.R. (1992). «Three Types of Risk Assessment and the Emergence of Port-Normal Science». En: KRIMSKY, S.; GolDING, D. (eds.). Social Theories of Risk. Westport, Londres: Praeger, p. 251-275.

MSC (MINISTERIO DE SANIDAD Y CONSUMO) (2001). Campos electromagnéticos y salud pública: Resumen informativo elaborado por el Ministerio de Sanidad y Consumo a partir del informe técnico realizado por el Comité de Expertos Independientes. Madrid: Ministerio de Sanidad y Consumo, Dirección General de Salud Pública y Consumo, Subdirección General de Sanidad Ambiental y Salud Laboral.

OSI (ObSERVATORI DE LA SOCIETAT DE LA INFORMACIÓ) (2001). Estadístiques de la Societat de la Informació Catalunya 2001. Barcelona: Secretaria de Telecomunicacions i Societat de la Informació. URL:http://www.gencat.es/dursi [consulta 15-032002].

PidgeOn, N.; KaSPERSON, R.E.; Slovic, P. (2003). The social amplification of risk. Cambridge: Cambridge University Press.

Slovic, P. (1987). «Perception of Risk». Science, núm. 236: 280-285. 
StARR, C. (1969). «Social Benefit versus Techonological Risck». Science, núm. 165: $1.232-1.238$.

VeCCHIA, P. (2001). "Campi elettromagnetici e salute: panorama delle conoscenze». Le onde elettromagnetiche: rischi e certeze. Atti del seminario su ELF e RF. San Marino: AIEP Editore, p. 171-184.

WynNE, B. (1992). «Misunderstood misunderstanding: Social Identities and Public Uptake of Science». Public Understanding of Science, 3, núm. 1: 281-304.

Wynne, B. (1996). «May the Sheep Safely Graze? A Reflexive View of the Expert-Lay Knowledge Divide». En: LASH, S.; SZERSZYNSKI, B.; WynNe, B. Risk, Environment and Modernity. Londres: Sage, p. 44-83. 